\title{
An Evaluation of the Activity of Histidine-Rich Glycoprotein on Differentiated Neutrophil-Like Cells from Human Cell Lines
}

\author{
Yukinori Yoshii, Hidenori Wake, ㄱoshito Nishimura, Kiyoshi Teshigawara, Dengli Wang, \\ and Masahiro Nishibori \\ Department of Pharmacology, Graduate School of Medicine, Dentistry, and Pharmaceutical Sciences, Okayama University, \\ Okayama, Japan
}

Received June 24, 2020; accepted September 10, 2020

\begin{abstract}
Histidine-rich glycoprotein (HRG) treatment ameliorated the survival rate of septic mice by suppressing excess immunothrombus formation. Although such findings suggested that HRG may be one of the most useful drugs for sepsis, obtaining a stable experimental system to standardize the HRG drug product is difficult to achieve using neutrophils isolated from volunteers. This is due to the short survival time and individual differences of human neutrophils. In the present study, we determined whether the differentiated neutrophil-like cell lines exhibited similar responses to HRG compared with human purified neutrophils. All-trans retinoic acid (ATRA) was employed to induce the differentiation of the human myeloid leukemia cell lines HL-60 and NB-4. Thereafter, the cells were treated with Hank's balanced salt solution, human serum albumin, or HRG. The effects of HRG on these cells were evaluated according to cell shape, microcapillary passage, reactive oxygen species (ROS) production, neutrophil extracellular traps (NETs) formation, the expression of activated
\end{abstract}

CD11b, and cell viability. HRG maintained the round shape of differentiated neutrophil-like cells, decreased the time required by cells to pass through the microcapillaries, and inhibited ROS production, NETs formation, and the expression of activated $\mathrm{CD} 11 \mathrm{~b}$ on the cell surface. Moreover, the cells could survive longer in the presence of HRG than the control. The ATRAinduced differentiated cell lines could be used as alternatives to neutrophils to investigate the effects of HRG on neutrophils. This method can thus be used as an essential standardization test in pharmaceutical development.

\section{SIGNIFICANCE STATEMENT}

Human neutrophils exhibit varying responses to histidine-rich glycoprotein (HRG); however, all-trans retinoic acid-induced differentiated neutrophil-like cell lines can be used as reliably proxies to investigate the effects of HRG on neutrophils. Additionally, these cell lines can be employed in the development of therapies for the treatment of sepsis.

\section{Introduction}

Histidine-rich glycoprotein (HRG) is a $75-\mathrm{kDa}$ glycoprotein characterized by many histidine residues and GHHPH repeat sequences, synthesized in and secreted from the liver (Koide et al., 1986; Borza et al., 1996; Poon et al., 2011). It exists in healthy human plasma at $60-100 \mu \mathrm{g} / \mathrm{ml}$ (approximately $1 \mu \mathrm{M}$ ) (Poon et al., 2011) and is reported to possess a variety of physiologic activities, including the suppression of erythrocyte aggregation (Zhong et al., 2018), vascular endothelium protection (Gao et al., 2019, 2020), and regulation of blood cell adhesion (Wake et al., 2016). In the animal experiments, we reported that serum HRG levels in septic mice with cecal

The research was supported by grants from the Japan Agency for Medical Research and Development (AMED) (19im0210109h0003) to M.N. and the Japan Society for the Promotion of Science (JSPS) Grant-in-Aid for Scientific Research (KAKENHI) (17K15580) to H.W. Human fresh-frozen plasma was kindly provided by the Japanese Red Cross Society.

https://doi.org/10.1124/jpet.120.000182. ligation and puncture markedly dcerased, whereas their survival rate increased after HRG supplementation, and thus HRG was identified as an effective therapeutic strategy for sepsis (Wake et al., 2016). In the clinical studies, it was suggested that plasma HRG level may be not only an excellent biomarker for patients who are septic but also a prediction factor for the prognosis of patients who are septic in an intensive care unit (Kuroda et al., 2018; Nishibori et al., 2018).

In 2016, a major revision to the definition of sepsis was performed. As a result, sepsis is now defined as a "lifethreatening organ damage due to uncontrolled host reactions to infections" (Singer et al., 2016). The global burden of sepsis has been increasing, which requires progressive and strategic treatment of patients with the life-threatening condition (Angus and van der Poll, 2013; Fleischmann et al., 2016; Rhee et al., 2017). However, there is no specific treatment of sepsis, which thereby contributes to its high mortality (Reinhart et al., 2017). Therefore, discovering an alternative biomarker and a therapeutic target for sepsis is urgently required

ABBREVIATIONS: AM, acetoxymethyl; AML, acute myelogenous leukemia; ATRA, all-trans retinoic acid; BSA, bovine serum albumin; cit-histone $\mathrm{H} 3$, citrullinated histone H3; dHL-60, differentiated neutrophil-like HL-60; dNB-4, differentiated neutrophil-like NB-4; FITC, fluorescein isothiocyanate; fMLP, $N$-formyl-L-methionyl-L-leucyl-phenylalanine; HBSS, Hank's balanced salt solution; HRG, histidine-rich glycoprotein; HSA, human serum albumin; MC-FAN, Microchannel array flow analyzer; NET, neutrophil extracellular trap; NBT, nitroblue tetrazolium; Ni-NTA, nickel-nitrilotriacetic acid; PMA, phorbol 12-myristate 13-acetate; ROS, reactive oxygen species; TBS, Tris-buffered saline. 
(Reinhart et al., 2012; Opal et al., 2014). We have already started the project to develop human plasma-derived HRG preparation for the treatment of sepsis. Thus, to standardize purified HRG as a biologic material, it is imperative to establish the convenient and reliable assay system for evaluating each lot of HRG prepared from pooled plasma that would be used as the treatment of patients who are septic.

In vitro experiments using human purified neutrophils in particular showed that the passage of neutrophils through a microvascular model was remarkably improved by HRG supplementation because it maintained the spherical neutrophil shape with smooth surface without microvilli and modified their adhesiveness without attachment to the vascular wall model (Wake et al., 2016). HRG also inhibited reactive oxygen species (ROS) production as well as neutrophil extracellular traps (NETs) formation from neutrophils and cell death (Wake et al., 2016). Collectively, HRG could maintain the quiescent state of neutrophils. The rapid decrease in plasma levels of HRG in septic mice might result in a loss of its regulatory effects on circulating neutrophils, leading to the attachment of neutrophils to vascular endothelial walls and the intravascular formation of immunothrombus.

Because the response of neutrophils to severe infections is closely related to the pathology of sepsis, identifying the effects of HRG on neutrophils is crucial. However, owing to the difficulty associated with the use of human neutrophils in experiments because of their short lifespan, the individual differences in neutrophils among donors, and the challenges to establish a stable experimental system, the establishment of standardized cells to estimate the effects of human plasmaderived HRG is desirable. Therefore, we examined the effects of HRG on the all-trans retinoic acid (ATRA)-induced differentiated neutrophil-like cells (NLCs) from HL-60 and NB-4 (Barber et al., 2008; Gupta et al., 2014). Herein, we aimed to determine whether the NLCs could be a reliable proxy for neutrophils in HRG- and sepsis-related researches.

\section{Materials and Methods}

Reagents. Nickel-nitrilotriacetic acid (Ni-NTA) agarose gel was obtained from Qiagen (Hilden, Germany). $N$-formyl-L-methionyl-Lleucyl-phenylalanine (fMLP) was obtained from Peptide Institute, Inc. (Minoh, Japan). ATRA, nitroblue tetrazolium (NBT), isoluminol, horseradish peroxidase type IV and zymosan A from Saccharomyces cerevisiae, and phorbol 12-myristate 13-acetate (PMA) were obtained from Sigma-Aldrich (St. Louis, MO). Hoechst33342, calcein-AM, and SYTOX orange were obtained from Life Technologies (Carlsbad, CA). Anti-histone $\mathrm{H} 3 \mathrm{Ab}$ (citrulline R17 + R2 + R8) [citrullinated histone H3 (cit-histone H3)] and Alexa Fluor 488-labeled anti-rabbit IgG Ab were obtained from Abcam (Cambridge, UK). Fluorescein isothiocyanate (FITC)-labeled anti-human activated CD11b Ab (clone: CBRM1/5) was obtained from eBioscience (San Diego, CA). Blocking One was obtained from Nacalai Tesque (Kyoto, Japan). Anti-human HRG monoclonal antibody (Rat) and anti-human HRG polyclonal antibody (Rabbit) were synthesized in our laboratory.

Isolation of Human Neutrophils from Human Peripheral Blood. Peripheral blood polymorphonuclear cells were purified from heatlhy donors using Polymorphprep density gradient media (Alere Technologies AS, Oslo, Norway). In line with the ethical guidelines of Okayama University, written informed consents were obtained from healthy volunteers $(n=5)$, and we drew blood from the cubital vein. In brief, heparinized peripheral blood was layered over Polymorphprep and centrifuged at $500 \mathrm{~g}$ for 35 minutes. Polymorphonuclear cells were then harvested and diluted with PBS. After centrifugation at $400 \mathrm{~g}$ for
10 minutes, the purity of neutrophils was confirmed to be more than 95\% under microscope. Subsequently, neutrophils were used at appropriate concentrations for each experiment.

HRG Purification from Human Plasma. HRG was purified from human plasma (obtained from the Japanese Red Cross Society) by affinity chromatographic methods using Ni-NTA, as previously described (Mori et al., 2003). Briefly, protease inhibitors were added to human plasma. Thereafter, the mixture was centrifuged twice at $10,000 \mathrm{rpm}$ at $4^{\circ} \mathrm{C}$ for 15 minutes. The supernatant was incubated with Ni-NTA agarose gel, which was pre-equilibrated with $10 \mathrm{mM}$ Tris-buffered saline (TBS) ( $\mathrm{pH} 8.0$ ) and suspended in the same buffer. After gently mixing for 2 hours at $4^{\circ} \mathrm{C}$, the gel suspension was transferred into a column, washed with $50 \mathrm{mM}$ imidazole in $10 \mathrm{mM}$ TBS (pH 8.0), $2 \mathrm{M} \mathrm{NaCl}$ in $10 \mathrm{mM}$ TBS ( $\mathrm{pH} 8.0$ ), and $100 \mathrm{mM}$ imidazole in $10 \mathrm{mM}$ TBS ( $\mathrm{pH}$ 8.0) to enable the passage of all unbound proteins through the column. Finally, HRG was eluted with $10 \mathrm{mM}$ TBS $(\mathrm{pH}$ 8.0) containing $500 \mathrm{mM}$ imidazole. After exchanging the buffer by dialysis, the eluate was loaded onto a Mono Q column (GE Healthcare, Little Chalfont, UK) and separated by an $\mathrm{NaCl}$ gradient. The purified HRG was identified and collected by sodium dodecyl sulfatepolyacrylamide gel electrophoresis using a Western blot with an anti-human HRG polyclonal antibody. The obtained human HRG was stored at $-20^{\circ} \mathrm{C}$ until use.

Differentiation of HL-60 and NB-4 by ATRA. The human acute myelogenous leukemia (AML) cell lines HL-60 (at the myeloblast stage of development, AML-M2) and NB-4 cells (at the promyelocyte stage of development, AML-M3) were obtained from RIKEN Cell Bank (RCB0041) and CLS Cell Lines Service (GmbH). The cells were cultured in RPMI-1640 medium (Sigma-Aldrich) supplemented with 10\% (v/v) FBS (Thermo Fisher Scientific, Waltham, MA), L-glutamine (Sigma-Aldrich), and penicillin streptomycin (Thermo Fisher Scientific) and maintained in a humidified $5 \% \mathrm{CO}_{2}$ environment at $37^{\circ} \mathrm{C}$. The media were routinely passaged and diluted every 3 to 4 days to maintain exponential growth. All experiments were performed with cells from passages between 10 and 25 .

ATRA (Sigma-Aldrich) was dissolved in $99.5 \%$ ethanol as a $10-\mathrm{mM}$ stock solution. The solution was stored at $-80^{\circ} \mathrm{C}$ until use. The final concentration of ethanol in the culture was maintained at less than $0.1 \% \mathrm{v} / \mathrm{v}$. HL-60 or NB-4 cells were diluted to $2.5 \times 10^{5}$ cells $/ \mathrm{ml}$ in RPMI-1640 culture medium supplemented with $10 \%$ FBS. ATRA was added to the HL-60 and NB-4 cell culture to yield the final concentrations of 10 and $1 \mathrm{mM}$, respectively. HL-60 and NB- 4 were passaged 2 or 3 days after the addition of ATRA. Live cells were isolated with Dead Cell Removal Kit per the manufacturer's protocol (Miltenyi Biotec Inc., Auburn, CA).

The NBT reduction test is one of the neutrophil function tests that examines the phagocytotic activity and function of neutrophil enzyme systems (Blair et al, 1985). This method was used as a functional measure of the maturation of HL- 60 and NB- 4 cells. A total of $1.0 \times 10^{6}$ cells was collected by centrifugation and resuspended in $0.25 \mathrm{ml}$ of RPMI-1640 medium (Sigma-Aldrich) supplemented with $10 \%(\mathrm{v} / \mathrm{v})$ FBS (Thermo Fisher Scientific). Subsequently, $0.25 \mathrm{ml}$ of $0.1 \%$ NBT with $5 \mu \mathrm{l}$ PMA was added to each sample, and the cell suspensions were incubated at $37^{\circ} \mathrm{C}$ for 30 minutes. Samples were then fixed by the addition of an equal volume of $2 \%$ paraformaldehyde in PBS. Cells were observed with a BZ-8000 microscope (Keyence Corporation, Osaka, Japan). If the cells were differentiated into neutrophils, the yellow NBT dye was internalized, thereby resulting in the formation of black formazan due to reduction. The stained and nonstained cells were counted manually under the microscope. Both differentiated neutrophil-like HL-60 cells (dHL-60) and differentiated neutrophillike NB-4 cells (dNB-4) showed stable differentiation rates (Fig. 1).

Observation of the Morphologic Changes in Differentiated Cells. To clarify the effects of HRG on the morphology of dHL-60 and dNB-4 cells, cell shape was observed by calcein-AM (for cytosol) and Hoechst33342 (for nuclei) staining as described previously (Wake et al., 2016). Thereafter, $100 \mu \mathrm{l}\left(5 \times 10^{4}\right.$ cells/well $)$ of the two cell lines and human neutrophils (as a positive control) were aliquoted into each 
A

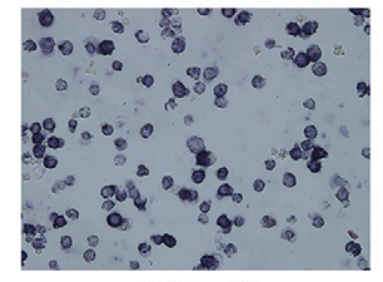

C
dHL-60

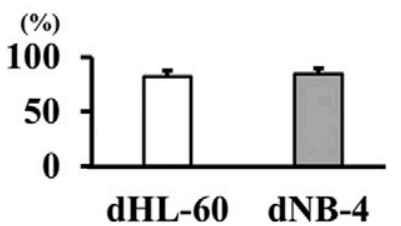

B

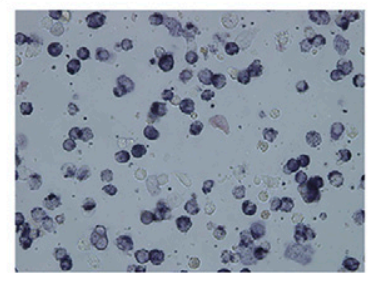

D

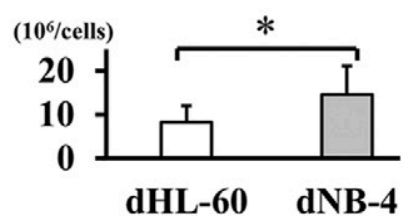

Fig. 1. Differentiation of the human myeloid cell lines to neutrophil-like cells. HL-60 and NB-4 are human AML cell lines that can be induced by ATRA to form mature neutrophil-like differentiated cells. (A and B) The NBT-stained dHL-60 and dNB-4 cells were induced for 5 days in 10 and $1 \mu \mathrm{M}$ of ATRA. (C) The ATRA-induced cell differentiation rates were determined by manually counting the NBT positive cells at original magnification, $10 \times$. The results are presented as the average of five different visual fields. Eighty to $90 \%$ of HL-60 and NB-4 cells were differentiated to neutrophil-like cells (dHL-60 and dNB-4 cells, respectively) by ATRA. (D) HL-60 or NB-4 cells were diluted to $2.5 \times 10^{5}$ cells $/ \mathrm{ml}$ (total $2.5 \times 10^{6}$ cells), and ATRA was added to the HL-60 and NB-4 cell culture. After the induction of cell differentiation, the absolute number of cells was counted. The results are the mean \pm S.D. of five experiments. $* P<0.05$.

polystyrene well. The cells were incubated with different concentrations of HRG (0.01-1 $\mu \mathrm{M})$ in the presence of control IgG or anti-HRG $\mathrm{Ab}$, human serum albumin (HSA) $(1 \mu \mathrm{M})$, bovine serum albumin (BSA) $(1 \mu \mathrm{M})$, or fMLP $(1 \mu \mathrm{M})$ at $37^{\circ} \mathrm{C}$ for 1 hour. The In Cell Analyzer 2000 System (GE Healthcare) was used for morphologic observation. The data were analyzed with the In Cell Investigator Version 1.62 (GE Healthcare). Additionally, the morphologic characteristics of cells were assessed by cell area (square micrometer per cell); the inclusion area per cell in the extended cytoplasm area was automatically determined using the In Cell Analyzer 2000 System.

Microchannel Array Flow Analyzer. The cell samples were prepared as described above. Briefly, the cells were incubated with either of the following reagents (HRG, HSA, BSA, fMLP: each at a final concentration $1 \mu \mathrm{M}$ ) at $37^{\circ} \mathrm{C}$ for 1 hour. Thereafter, the passage of cells through the microcapillary slits $(7.0-\mu \mathrm{m}$ width, $4.5-\mu \mathrm{m}$ depth) was evaluated by Microchannel array flow analyzer (MC-FAN) (MC Laboratory, Japan). Briefly, the cells flowed through the siliconbased artificial microchannels under a constant suction of $-20 \mathrm{~cm}$ $\mathrm{H}_{2} \mathrm{O}$. Thereafter, the passage time of the $100-\mu l$ sample through the microcapillaries was determined. The passage time was dependent on both the cell size and the cell adhesiveness, and the cells adhered to the silicon-coated microchannels based on their adhesiveness.

Expression Analysis of Activated CD11b on the Cell Surface by Flow Cytometry. Both dHL-60 and dNB-4 samples were stimulated with $100 \mathrm{ng} / \mathrm{ml}$ of PMA in the presence of one of the reagents [Hank's balanced salt solution (HBSS), HSA, or HRG, each at a concentration of $1 \mu \mathrm{M}$ ] for 1 hour at $37^{\circ} \mathrm{C}$ in a $5 \% \mathrm{CO}_{2}$ atmosphere. Thereafter, the cells were stained with FITC-labeled anti-human activated CD11b antibody for 25 minutes at $4^{\circ} \mathrm{C}$ and fixed with $0.5 \%$ paraformaldehyde in PBS for 30 minutes at $4^{\circ} \mathrm{C}$. The extent of activated CD11b expression in cells was analyzed using MACSQuant Analyzer (Miltenyi Biotec, Bergisch Gladbach, Germany).

Determination of Extracellular ROS Production. Because of the short half-life and reactivity of ROS, direct observation of the amount of ROS production is challenging. Under the presence of ROS, horseradish peroxidase type IV oxidizes isoluminol and induces luminescence. Our method harnessed the reaction and observed the amount of ROS production indirectly. Briefly, isoluminol (final concentration, $50 \mu \mathrm{M}$ ) and horseradish peroxidase type IV (final concentration, $4 \mathrm{U} / \mathrm{ml}$ ) were added to the dHL- 60 and $\mathrm{dNB}-4$ cell suspensions. Thereafter, the suspensions $\left(100 \mu \mathrm{l} ; 5 \times 10^{5}\right.$ cells $)$ were aliquoted into a 96-well plate (BD Biosciences). Subsequently, HRG, HSA (each at a final concentration of $1 \mu \mathrm{M}$ ), or HBSS was added to each well. In addition, the zymosan suspension (final concentration of $100 \mathrm{ng} / \mathrm{ml}$ ) was simultaneously added to each well. Extracellular ROS production was measured 10 times (every 5 -minute interval) from the beginning of incubation at $37^{\circ} \mathrm{C}$ by detecting chemiluminescence intensity using Flexstation 3 (Molecular Devices, Sunnyvale, CA).

Observation of NET Formation. Citrullination of histone H3 and $\mathrm{H} 4$ by peptidylarginine deaminase 4 is required for chromatin decondensation, which is an essential step for NET formation. We observed NET formation using cit-histone H3 with immunocytochemical analysis. The dHL-60 and dNB-4 cells were prepared, as mentioned above. For dead cell imaging, the cells were treated in advance with a cell-impermeable DNA fluorescent dye ( $5 \mu \mathrm{M}$ SYTOX orange). PMA $100 \mathrm{ng} / \mathrm{ml}$ and one of the reagents (HBSS, HSA, or HRG, each at concentration of $1 \mu \mathrm{M})$ were added to the cell suspensions. Thereafter, the cell suspensions $\left(100 \mu \mathrm{l} ; 5 \times 10^{5}\right.$ cells $)$ were aliquoted into a 96 -well plate (BD Biosciences). The cells were incubated for 2 (neutrophils) or 4 hours (dHL-60 and dNB- 4 cells) at $37^{\circ} \mathrm{C}$ in $5 \% \mathrm{CO}_{2}$ to stimulate NETs formation. Subsequently, the supernatant was gently removed, and the cells were fixed with $4 \%$ of paraformaldehyde for 20 minutes at $4^{\circ} \mathrm{C}$. To evaluate NET formation by immunocytochemical analysis, the samples were permeabilized with $0.1 \%$ Triton X-100 in TBS for 1 minute and blocked with Blocking One for 10 minutes at room temperature. After the addition of the primary antibody (anticit-histone $\mathrm{H} 3 \mathrm{Ab}$ at $10 \mu \mathrm{g} / \mathrm{ml}$ ) to the samples, the samples were incubated for 2 hours at room temperature. After three rounds of washing with TBS, the secondary antibody (Alexa Fluor 488labeled anti-rabbit IgG at $2 \mu \mathrm{g} / \mathrm{ml}$ ) was added to the samples, and this was followed by incubation for 1 hour at room temperature. Dead cells (SYTOX orange positive site) and NET formation (citHiston H3 positive site) were observed using the In Cell Analyzer 2000 System.

Cell Viability. Purified human neutrophils, dHL-60 cells, and dNB-4 cells were prepared, as mentioned above. To evaluate cell viability, live cells were stained by calcein-AM (for cytosol) for 15 minutes at $37^{\circ} \mathrm{C}$ in $5 \% \mathrm{CO}_{2}$. PMA $100 \mathrm{ng} / \mathrm{ml}$ was added to the cell suspensions in the presence of one of the reagents (HBSS, HSA, or $\mathrm{HRG}$, each at a concentration of $1 \mu \mathrm{M})$. Each suspension $(100 \mu \mathrm{l} ; 5 \times$ $10^{5}$ cells) was aliquoted into a 96-well plate (BD Biosciences) and incubated for 8 hours at $37^{\circ} \mathrm{C}$ in $5 \% \mathrm{CO}_{2}$. Cell viability was defined as the percentage of live cells at 8 hours postincubation. The number of live cells was calculated using the In Cell Analyzer 2000 System based on the presence of calcein-AM staining.

Statistical Analysis. Statistical significance was evaluated by Student's $t$ test for comparisons between two groups or Tukey's test for multiple comparison. All data are presented as the mean value \pm S.D. The $P$ values less than 0.05 were considered to be statistically significant. The analyses were conducted with SPSS software version 24 (SPSS, Chicago, IL).

\section{Results}

Effects of HRG on the Morphology of dHL-60 and dNB-4 Cells. The dHL-60 or dNB-4 cells prestained with calcein-AM (for cytosol) and Hoechst33342 (for nuclei) were observed using the In Cell Analyzer 2000 System. The morphologic characteristics of cells were assessed according to the cell area. HRG reduced the cell volume of dHL-60 and dNB-4 and the purified human neutrophils in a dosedependent manner and exhibited a significant effect at 
concentrations $\geq 0.03 \mu \mathrm{M}$ (Fig. $2, \mathrm{~A}$ and B). When $1 \mu \mathrm{M}$ of HRG was assumed to cause the maximal effects, the $\mathrm{EC}_{50}$ of $\mathrm{HRG}$ that caused the less-surface-area effect on dHL-60, dNB-4, and neutrophils was $0.046,0.060$, and $0.056 \mu \mathrm{M}$, respectively. These effects of HRG were not observed with HSA, BSA, and fMLP (Fig. 2, C and D). Additionally, HRG significantly reduced the cell surface area in both cell lines and human neutrophils (Fig. 2, C and D). The effect of HRG on dNB-4 was inhibited by the addition of three types of anti-HRG antibodies and human neutrophils. However, in dHL-60, there was a significant reversal in the effect of HRG by the addition of the mouse monoclonal anti-HRG antibody (IgG2b) (Fig. 2, E-H).
HRG Decreased the Adhesion Property of dHL-60 and dNB-4 Cells. To examine the effects of HRG on the adhesion property of dHL-60 and dNB-4, the passage of cells through the microcapillary was observed using MC-FAN. Owing to the abundant cytoplasm and high adhesiveness, cells without HRG treatment did not smoothly pass through the slits. Moreover, the flow path was often completely blocked around the slits (red arrowhead) (Fig. 3A). In the group treated with HRG $(1 \mu \mathrm{M})$, the cells could pass through the microcapillaries at a significantly faster rate than the other groups (Fig. 3B). Subsequently, we determined whether HRG $(1 \mu \mathrm{M})$ inhibited the activated-CD11b expression on the surface of cells by flow cytometry. The extent of activated-CD11b expression on
A

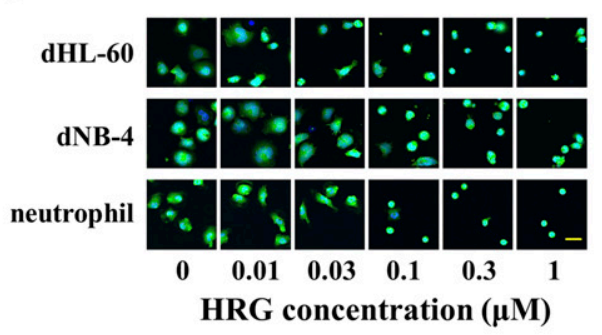

C

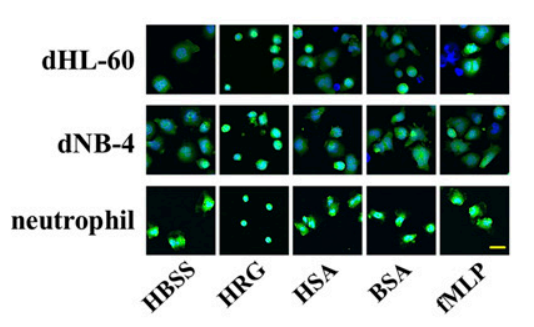

E

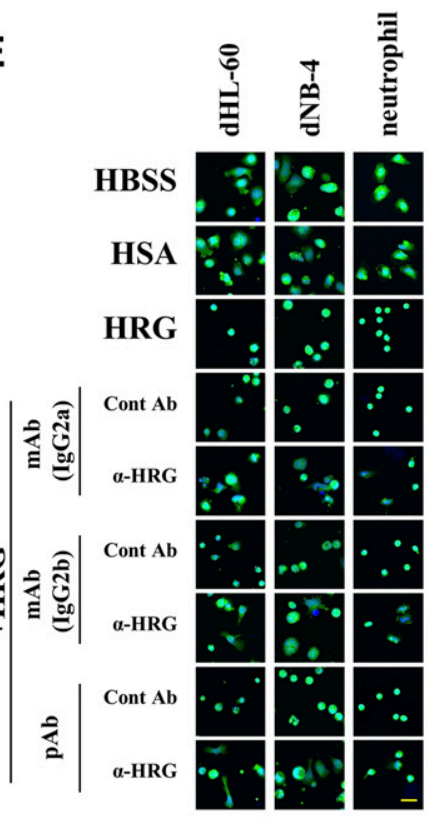

$\mathbf{F}$

G

H
B

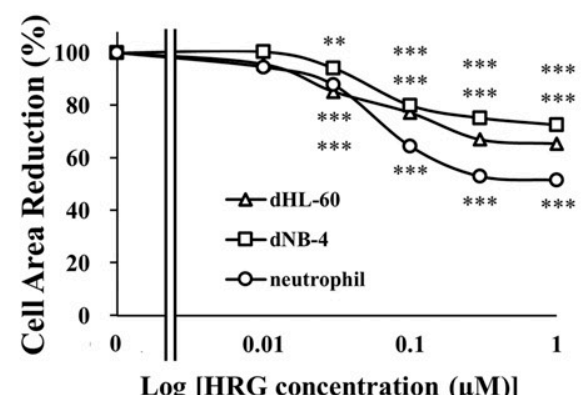

D
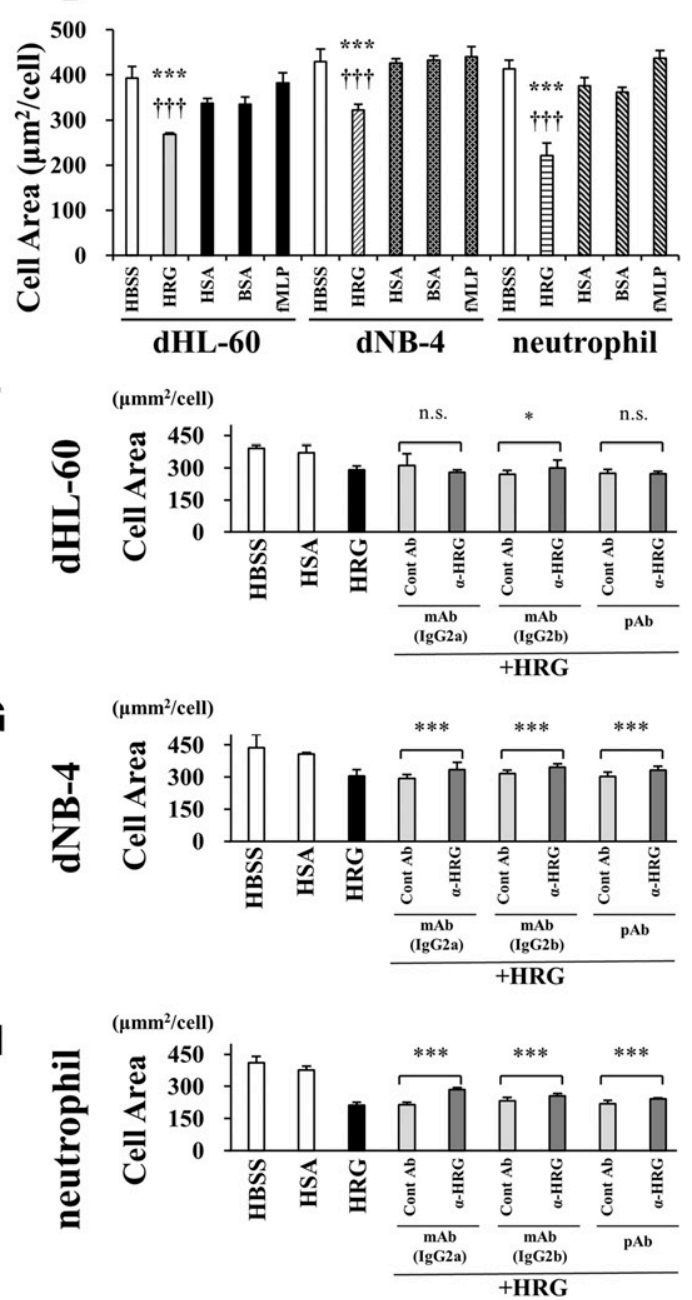

Fig. 2. The effect of HRG on neutrophillike cell surface area. The shape of the differentiated cells and purified human neutrophils was analyzed after staining of the cell nuclei and cytosol with Hoechst33342 and calcein-AM, respectively, for 15 minutes. Changes in cell morphology were observed with the In Cell Analyzer 2000 System. The data were analyzed with In Cell Investigator Version 1.62. (A) The cells were incubated with $0.01-1 \mu \mathrm{M}$ of HRG. Scale bar, $20 \mu \mathrm{m}$. (B) Cell Area (square micrometer per cell) represents the inclusion area per cell in the extended cytoplasm area. HRG caused a change in shape to spherical and inhibited the irregularity and expanding of the cell shape of dHL- 60 and dNB-4 cells and the human neutrophils according to the concentration. The results are the mean \pm S.D. of 12 experiments. $* P<$ $0.05 ; * * P<0.01 ; * * * P<0.001$ vs. HBSS. (C) The cells were incubated with one of the reagents at the same concentration of $1 \mu \mathrm{M}$ for comparison. (D) The spherical shape-inducing effects of HRG were evident. The results are the mean \pm S.D. of 12 experiments. $* * * P<0.001$ vs. HBSS, ${ }^{\dagger \dagger} P<0.001$ vs. HSA. Scale bar, 20 $\mu \mathrm{m}$. (E) The cells were incubated with HSA (1 $\mu \mathrm{M})$, HRG (1 $\mu \mathrm{M})$, HRG, and control IgG or anti-HRG $\mathrm{Ab}(10 \mu \mathrm{g} / \mathrm{ml})$ for 1 hour. The anti-HRG monoclonal/polyclonal $\mathrm{Ab}$ could significantly inhibit the spherical shape-inducing effects of HRG. (F-H) In the NB4 cell line, the change to a spherical shape was reversed by all antibodies and neutrophils. The results are the mean \pm S.D. of 12 experiments. n.s. not significant; $* P<0.05 ; * * P<0.01$; $* * * P<0.001$. Scale bar, $20 \mu \mathrm{m}$. 
A

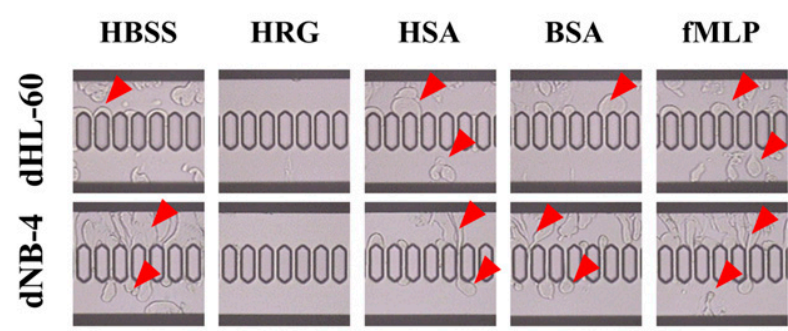

B

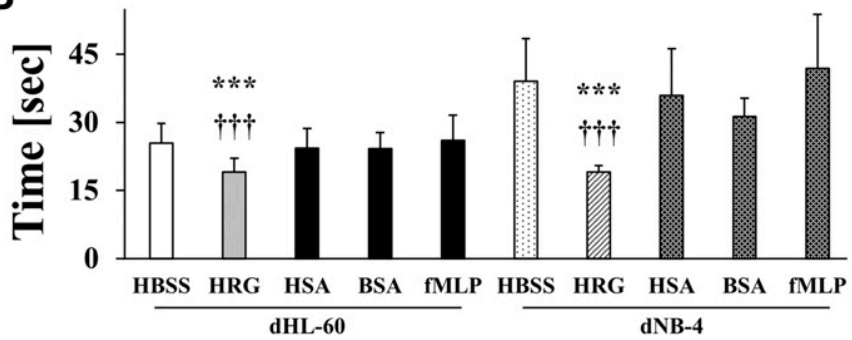

Fig. 3. HRG ameliorated microcapillary passage of neutrophil-like cells. Cell incubation was initiated with one of the reagents (HRG, HSA, BSA, fMLP: each at a final concentration of $1 \mu \mathrm{M}$ ) at $37^{\circ} \mathrm{C}$ for 60 minutes. Thereafter, the cells were applied to an MC-FAN and allowed to flow through silicon-based artificial microchannels under a constant suction of $-20 \mathrm{~cm} \mathrm{H}_{2} \mathrm{O}$. (A) Red arrowheads indicate the adhesion of cells to the microcapillary during the experiment. (B) The passage time of the $100-\mu l$ samples through microcapillaries was determined. The results are the mean \pm S.D. of six experiments. $* * * P<0.001$ vs. HBSS, ${ }^{\dagger \dagger} P<0.001$ vs. HSA.

cells was analyzed using the MACSQuant Analyzer. HRG significantly decreased the cell surface activated-CD11b expression on dHL-60 (Fig. 4). Additionally, HRG significantly reduced the activated-CD11b expression on dNB-4 compared with the HBSS control. However, the effect was not statistically significant compared with the HSA group $(P=0.076)$.

HRG Inhibited ROS Production and NET Formation in dHL-60 and dNB-4. To determine the production of ROS by the two differentiated cell lines, we measured the chemiluminescence produced by the oxidation of isoluminol. Because the differentiated NLCs cannot produce ROS without additional stimulation, zymosan was employed to induce ROS production. Thus, $10 \mathrm{mg} / \mathrm{ml}$ of the zymosan suspension was added in the presence of HRG $(1 \mu \mathrm{M})$, HSA $(1 \mu \mathrm{M})$, or HBSS to both dHL-60 and dNB-4. After 20 minutes of incubation at $37^{\circ} \mathrm{C}$, extracellular ROS production was evaluated. Figure 5 demonstrates the significant inhibition of zymosan-induced ROS production in dHL-60 and dNB-4 caused by HRG compared with that observed in the other groups. To assess the effect of HRG on NET formation, PMA, a chemical inducer of NET formation, was added to the dHL-60 and dNB-4 cells prestained with SYTOX orange (cell-impermeable DNA fluorescent dye), in the presence of one of the reagents (HBSS, HSA, or HRG, each at a concentration of $1 \mu \mathrm{M})$. Thereafter, the cells were incubated either for 2 (neutrophils) or for 4 hours (dHL-60 and dNB-4 cells) at $37^{\circ} \mathrm{C}$ in $5 \% \mathrm{CO}_{2}$ on a plastic 96 -well plate. NET formation was evaluated as a cit-histone $\mathrm{H} 3$ positive site by immunocytochemical analysis on the In Cell Analyzer 2000 System with the cell area. Compared with the HBSS and HSA groups, HRG significantly inhibited NET formation in both dHL-60 and dNB-4 cells (Fig. 6).
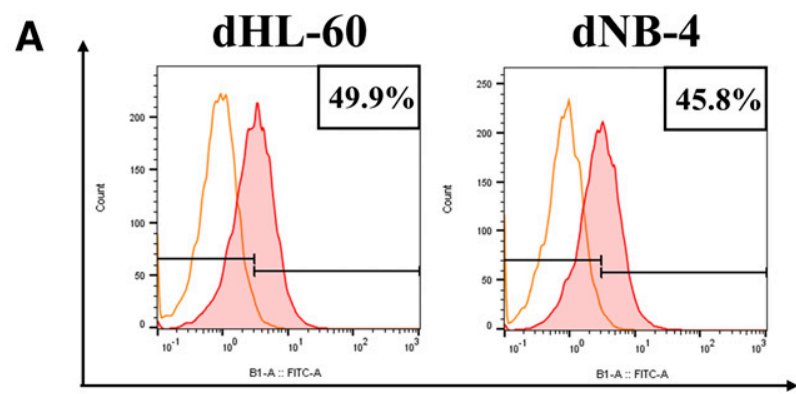

B

Activated CD11b positive

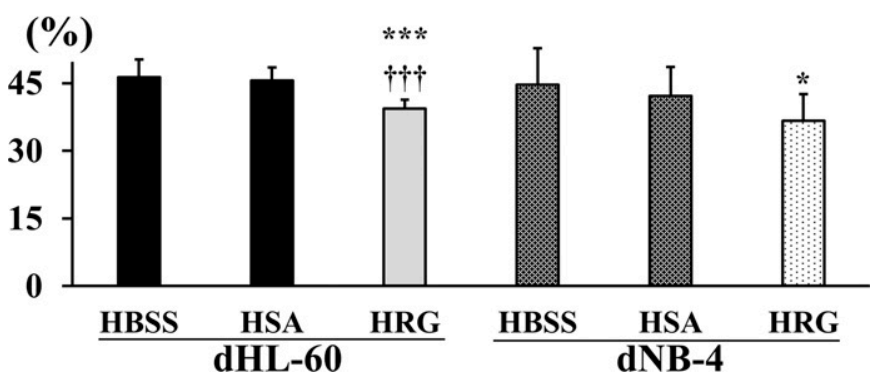

Fig. 4. The expression level of CD11b on the surface of neutrophil-like cells decreased after HRG treatment. The dHL-60 or dNB-4 cells were stimulated with $100 \mathrm{ng} / \mathrm{ml}$ of PMA for 1 hour at $37^{\circ} \mathrm{C}$ in a $5 \% \mathrm{CO}_{2}$ atmosphere. After the cells were stained with FITC-labeled control Ab or FITC-labeled anti-human activated CD11b Ab for 25 minutes at $4^{\circ} \mathrm{C}$, flow cytometric analysis was performed with the MACSQuant Analyzer. (A) Figures indicate the process used to determine the number of activated CD11b-positive cells under PMA stimulation. Representative HBSS group images of the flow cytometric analysis were used to determine activated CD11b expression in the neutrophil-like cells stimulated with PMA. (B) The dHL-60 or dNB-4 cells were stimulated with $100 \mathrm{ng} / \mathrm{ml}$ of PMA in the presence of HRG $(1 \mu \mathrm{M})$, HSA $(1 \mu \mathrm{M})$, or HBSS for 1 hour at $37^{\circ} \mathrm{C}$ in a $5 \% \mathrm{CO}_{2}$ atmosphere. The results are expressed as mean \pm S.D. of nine experiments. $* P<0.05$ vs. HBSS, $* * * P<0.001$ vs. HBSS, ${ }^{\dagger \dagger} P<0.001$ vs. HSA.

dHL-60 and dNB-4 Survived Longer than Purified Human Neutrophils, and HRG Extended Their Survival. After the dHL-60 or dNB-4 cells were prestained with calcein-AM (for cytosol) and Hoechst33342 (for nuclei), the number of live cells was counted using the In Cell Analyzer 2000 System. Neutrophils viability was significantly lower

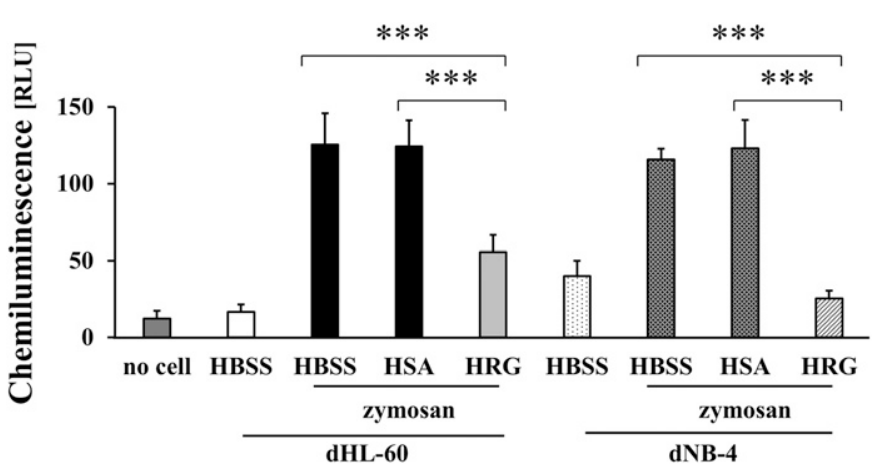

Fig. 5. HRG inhibited extracellular ROS production in neutrophil-like cells. The dHL-60 or dNB-4 cells were stimulated with $100 \mu \mathrm{g} / \mathrm{ml}$ of PMA in the presence of HRG $(1 \mu \mathrm{M})$, HSA $(1 \mu \mathrm{M})$, or HBSS. Thereafter, extracellular ROS production was determined using isoluminol as a substrate. The intensity of the chemiluminescence in the medium was evaluated by Relative light unit (RLU) using Flexstation 3. HRG significantly decreased ROS production after 20 minutes of incubation at $37^{\circ}$ C. The results are the mean \pm S.D. of five experiments. ${ }^{* * *} P<0.001$. 
A

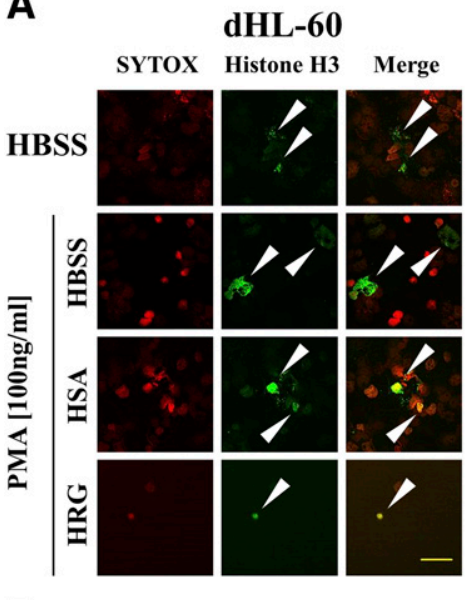

B

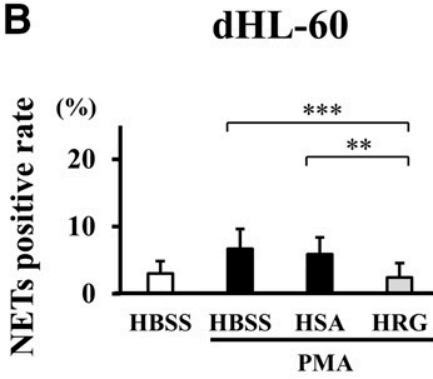

C

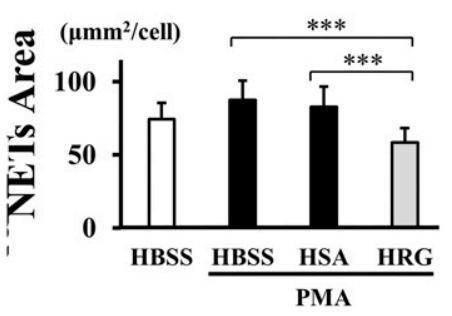

dNB-4

SYTOX Histone H3
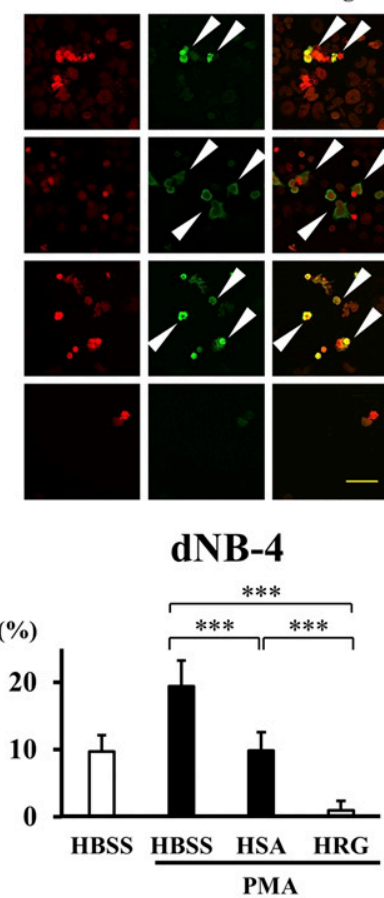

dNB-4

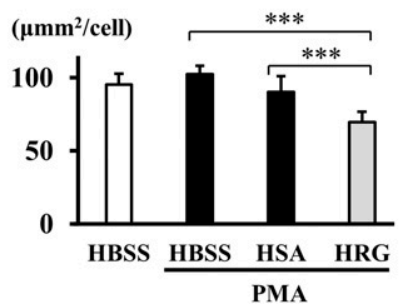

neutrophil

SYTOX Histone H3 Merge
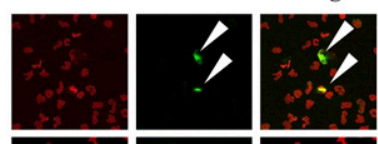

$\therefore 8$
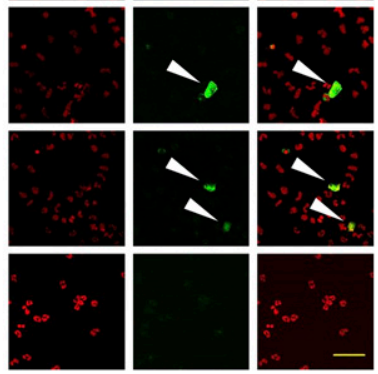

neutrophil

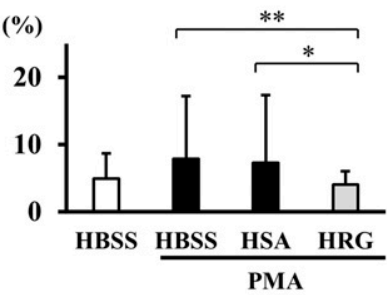

neutrophil

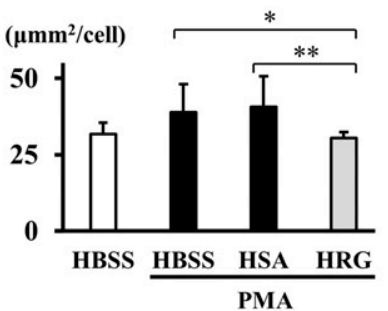

Fig. 6. HRG inhibited NET formation in neutrophil-like cells. The cells were stimulated with $100 \mu \mathrm{g} / \mathrm{ml}$ of PMA in the presence of HRG $(1 \mu \mathrm{M})$, HSA $(1 \mu \mathrm{M})$, or HBSS for 2 (neutrophil) or 4 hours (dHL60 and $\mathrm{dNB}-4)$. Thereafter, the cells were stained with SYTOX Orange (nuclei: orange) and anti-cit-histone $\mathrm{H} 3$ antibody (NETs: green) for fluorescence detection. (A) White arrowheads indicate NETs. Scale bar, $50 \mu \mathrm{m}$. (B and C) NETs were objectively evaluated with the rate of cithistone $\mathrm{H} 3$ positive cell number (\%) and cell area (square micrometer per cell) using the In Cell Analyzer 2000 System. HRG inhibited NET formation. The results are the mean \pm S.D. of 12 experiments. $* P<0.05 ; * * P<0.01 ; * * * P<0.001$.

than that of dHL-60 and dNB-4 at 8 hours after incubation (Fig. 7A).

PMA was added in the presence of the reagents (HBSS, HSA, HRG, each at a concentration of $1 \mu \mathrm{M}$ ) to cells for an 8 -hour incubation at $37^{\circ} \mathrm{C}$ in $5 \% \mathrm{CO}_{2}$. PMA stimulation markedly reduced the viability of all three cells; however, the addition of $\mathrm{HRG}$ significantly prolonged the survival time of every cell type under PMA stimulation (Fig. 7, B-D).

\section{Discussion}

The promyelocytic leukemia cell lines HL-60 and NB-4 are known to differentiate into neutrophil- or monocyte-like cells after induction by chemical stimuli (Idres et al., 2001; Jasek et al., 2008; Gupta et al., 2014). ATRA has been used to induce the differentiation of these cell lines into NLCs, which are often characterized by the NBT reduction test (Blair et al., 1985). In the present study, we obtained more than $80 \%$ NBTpositive cells from both HL-60 and NB-4 when the cells were exposed to 10 and $1 \mu \mathrm{M}$ of ATRA for 5 days. Thus, the procedure was simple and reliable. Additionally, it repeatedly yielded a considerable number of NLCs. In previous studies (Wake et al., 2016; Gao et al., 2019), HRG was observed to

induce the round shape of neutrophils, their smooth surface, and few microvilli. These neutrophils could easily pass through the microchannels and cause the lowest levels of spontaneous ROS production and less adhesion to the vascular endothelial cells and the artificial polystyrene wear. Therefore, we determined whether NLCs differentiated from HL60 and NB-4 by ATRA exhibited similar responses to HRG to evaluate the usefulness of the differentiated cells in the standardized regulation tests for the purified HRG materials from plasma.

One of the major effects of HRG on neutrophils involves the induction of a round shape with a smaller diameter and a smooth cellular surface as revealed by scanning electron microscopy. Under septic conditions, neutrophils are exposed to various immunochemical stimuli, which leads to cellular enlargement. Neutrophil enlargement is a result of overactivation, in which they are unable to function properly. These overactivated cells have excessive cell adhesiveness to microcapillaries, leading to immunothrombus formation and subsequent worse clinical outcomes in patients who are septic (Wake et al., 2016). In the present study, we observed similar concentration-dependent morphologic effects of HRG on the differentiated NLCs from HL-60 and NB-4. However, these 
A
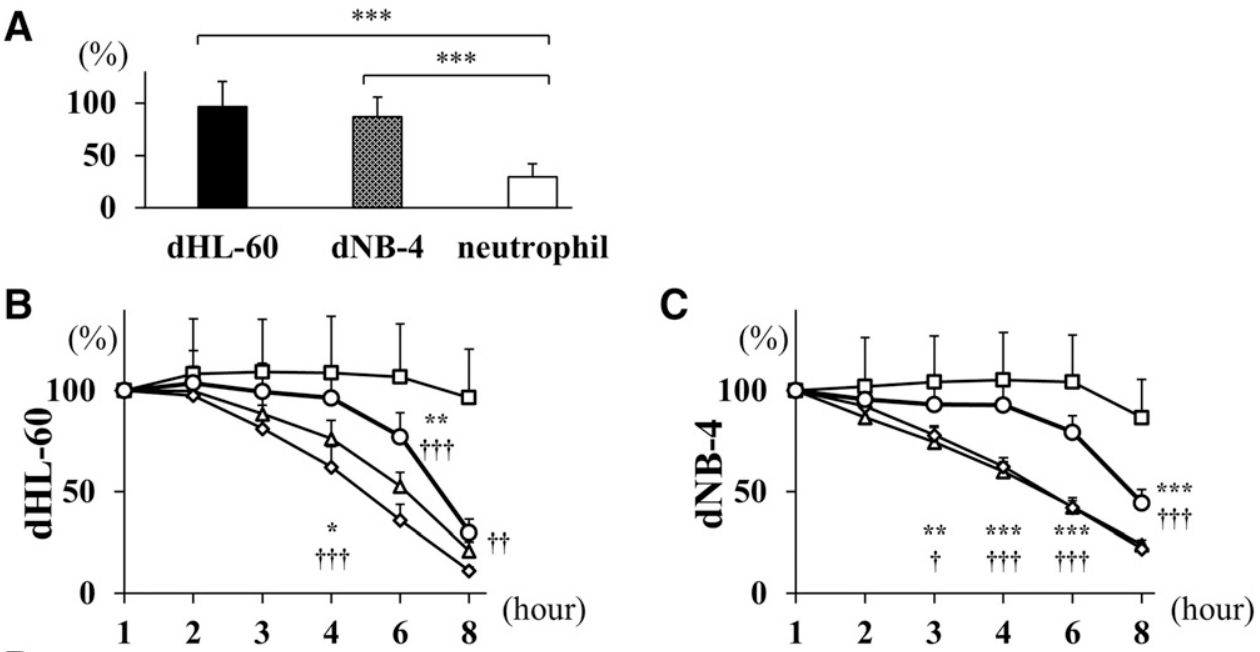

Fig. 7. Neutrophil-like cells can survive longer than purified human neutrophils, and the addition of HRG can extend cell survival rates. The dHL-60 and dNB-4 cells and purified human neutrophils were prestained with calcein-AM (for Cytosol). Thereafter, the cells were incubated with HBSS or $100 \mu \mathrm{g} / \mathrm{ml}$ of PMA in the presence of HRG $(1 \mu \mathrm{M})$, HSA $(1 \mu \mathrm{M})$, or HBSS for 8 hours at $37^{\circ} \mathrm{C}$ in a $5 \% \quad \mathrm{CO}_{2}$ atmosphere. The live cells (calcein positive sites) in each group were counted with the In Cell Analyzer 2000 System. (A) The dHL-60 and dNB-4 cells survived longer than purified human neutrophils after 8 hours of incubation in the unstimulated condition. The results are the mean \pm S.D. of 12 experiments. **** $P<0.001$ (B-D) PMA stimulation decreased cell survival rates; however,

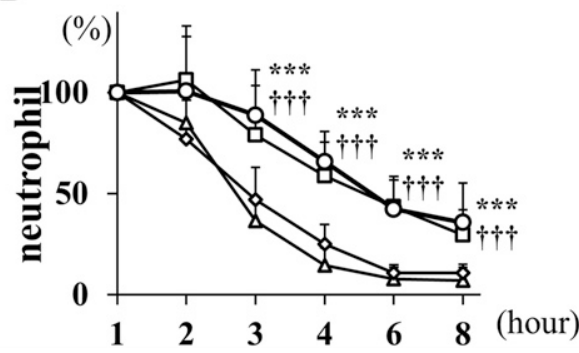

$$
\begin{aligned}
& \multimap-\text { HBSS } \\
& -\triangle \text { PMA + HBSS } \\
& \multimap-\text { PMA + HSA } \\
& \multimap-\text { PMA + HRG }
\end{aligned}
$$
HRG significantly improved cell viability compared with the other reagents after 6 hours of incubation, even with PMA stimulation. The results are the mean \pm S.D. of 12 experiments. $* P<0.05$; $* * * P<$ 0.001 vs. PMA + HBSS, ${ }^{\dagger} P<0.05 ;{ }^{\dagger \dagger} P<$ $0.01 ;{ }^{\dagger \dagger} P<0.001$ vs. PMA + HSA.

effects were inhibited by the addition of the antibody against HRG, thereby indicating the specificity of the effect of HRG. The morphologic effects of HRG on neutrophils and NLCs were comparable according to the potencies of HRG on each cell as determined with the concentration-effect curves. The $\mathrm{EC}_{50}$ values for neutrophils, HL-60-derived cells, and NB4-derived cells were $0.056,0.046$, and $0.060 \mu \mathrm{M}$, respectively, suggesting a similar mechanism of action of HRG on human neutrophil and NLCs. The most distinctive physiologic effect of HRG is considered to be the spherical shape-inducing effect, and in our laboratory, we assessed the quality of purified HRG with the extent of spherical shape-inducing effects on neutrophils as well as a Western blotting. Notably, the effective concentrations of HRG on human vascular endothelial cells were within the same range (Gao et al., 2019). HRG inhibited the PMA-induced upregulation of the expression of the activation epitope on CD11b on NLCs, as observed in neutrophils (Wake et al., 2016).

Because the activation of CD11b is related to the functional control of cellular adhesion through lymphocyte functionassociated antigen-1 on neutrophils, NLCs might receive functional regulation by HRG. Although NLCs have a larger size than human neutrophils, measuring the time taken by NLCs to pass through the microchannels of MC-FAN revealed that treatment with HRG reduced the time required for the passage of $100 \mu \mathrm{l}$ of cell suspension compared with those treated with other proteins. Furthermore, HRG significantly prolonged the survival time of NLCs and neutrophils under the stimulation of PMA. Excessive immunothrombus formation secondary to neutrophil intravascular cell adhesion detonates multiorgan dysfunction in patients who are septic, leading to high mortality. Previous studies suggested that HRG reduced the immunothrombus formation (Wake et al.,
2016) and reduced HRG concentration in patients who were severely septic (Kuroda et al., 2018). Altogether, analyzing neutrophil flow provides insight into the neutrophil function in sepsis, and these results suggest that NLCs responded to HRG in a very similar manner to human neutrophils.

Recent studies suggest that the pathophysiological cascade in septic conditions may include excessive ROS production in neutrophils, which is associated with NETosis, leading to the formation of immunothrombi. Although NETosis was initially characterized as a barrier system to prevent the diffusion of invasive bacteria, more global pathophysiological roles have been suggested (Brinkmann et al., 2004; Papayannopoulos et al., 2010; Phillipson and Kubes, 2011; Remijsen et al., 2011) The intravascular events that simultaneously damage the vascular endothelial cells should exacerbate microcirculation in the tissues and quickly produce multiple organ failure and disseminated intravascular coagulation. Herein, we used NLCs to demonstrate the NETosis-inhibiting effects of HRG. Thus, NLCs could be used to understand the mechanisms of NETosis and the effects of HRG on this process.

This study had some limitations. First, although standardized NLCs were produced herein, the reproducibility of the experiment has not been confirmed in other laboratories. Second, the responsiveness of the NLCs to HRG may not be identical to that of neutrophils because NLCs are derived from the differentiation of the human myeloid leukemia cell lines. Third, the reactivity of NLCs might be subject to cultural and differentiation-inducing conditions. Despite the limitations with NLCs, several issues arise when the mechanism used by HRG to modulate the shape or function of human neutrophils is investigated. First, human neutrophils are very short-lived and have a circulating half-life of $<8$ hours (Summers et al., 2010). As shown in Fig. 7D, isolated neutrophils also have 
a very short life span in the culture media. Second, there might be individual variations in survival time and responsiveness after isolation. Thus, it is impractical to conduct regulatory tests with isolated human neutrophils to evaluate the activity of purified HRG as a candidate drug for the treatment of sepsis (Wake et al., 2016; Kuroda et al., 2018; Nishibori et al., 2018). However, NLCs from HL-60 and NB-4 may be competent replacements for this purpose. Additionally, differences in the control test response and responsiveness to HRG were identified between NLCs derived from HL-60 and NB-4.

In the present study, ATRA was employed to induce the differentiation of the human myeloid leukemia cell lines HL60 and NB-4 into NLCs. Based on our findings, the differentiated NLCs had very similar reactivity to HRG relative to human neutrophils. Additionally, a stable experimental system could be easily obtained. Altogether, the methods developed herein to acquire the differentiated NLCs could be an essential tool not only for researchers but also for pharmaceutical developers to drive the sustainable drug-discovery efforts for sepsis treatment.

\section{Acknowledgments}

We thank the members of Central Research Laboratory, Okayama University Medical School, for enabling us to use their instruments: MACSQuant Analyzer and Flexstation 3.

\section{Authorship Contributions}

Participated in research design: Yoshii, Wake, Teshigawara, Wang, Nishibori.

Conducted experiments: Yoshii.

Performed data analysis: Yoshii, Wake.

Wrote or contributed to the writing of the manuscript: Yoshii, Wake, Nishimura, Nishibori.

\section{References}

Angus DC and van der Poll T (2013) Severe sepsis and septic shock. N Engl J Med 369:840-851.

Barber N, Belov L, and Christopherson RI (2008) All-trans retinoic acid induces different immunophenotypic changes on human HL60 and NB4 myeloid leukaemias. Leuk Res 32:315-322.

Blair OC, Carbone R, and Sartorelli AC (1985) Differentiation of HL-60 promyelocytic leukemia cells monitored by flow cytometric measurement of nitro blue tetrazolium (NBT) reduction. Cytometry 6:54-61.

Borza DB, Tatum FM, and Morgan WT (1996) Domain structure and conformation of histidine-proline-rich glycoprotein. Biochemistry 35:1925-1934.

Brinkmann V, Reichard U, Goosmann C, Fauler B, Uhlemann Y, Weiss DS, Weinrauch Y, and Zychlinsky A (2004) Neutrophil extracellular traps kill bacteria. Science 303:1532-1535.

Fleischmann C, Scherag A, Adhikari NK, Hartog CS, Tsaganos T, Schlattmann P, Angus DC, and Reinhart K; International Forum of Acute Care Trialists (2016) Assessment of global incidence and mortality of hospital-treated sepsis. Current estimates and limitations. Am J Respir Crit Care Med 193:259-272.

Gao S, Wake H, Gao Y, Wang D, Mori S, Liu K, Teshigawara K, Takahashi H, and Nishibori M (2019) Histidine-rich glycoprotein ameliorates endothelial barrier dysfunction through regulation of NF-кB and MAPK signal pathway. $B r$ $J$ Pharmacol 176:2808-2824.

Gao S, Wake H, Sakaguchi M, Wang D, Takahashi Y, Teshigawara K, Zhong H, Mori $\mathrm{S}$, Liu K, Takahashi H, et al. (2020) Histidine-rich glycoprotein inhibits highmobility group box-1-mediated pathways in vascular endothelial cells through CLEC-1A. iScience 23:101180.

Gupta D, Shah HP, Malu K, Berliner N, and Gaines P (2014) Differentiation and characterization of myeloid cells. Curr Protoc Immunol 104:22F.5.1-22F.5.28.

Idres N, Benoît G, Flexor MA, Lanotte M, and Chabot GG (2001) Granulocytic differentiation of human NB4 promyelocytic leukemia cells induced by all-trans retinoic acid metabolites. Cancer Res 61:700-705.

Jasek E, Mirecka J, and Litwin JA (2008) Effect of differentiating agents (all-trans retinoic acid and phorbol 12-myristate 13-acetate) on drug sensitivity of HL60 and NB4 cells in vitro. Folia Histochem Cytobiol 46:323-330.

Koide T, Foster D, Yoshitake S, and Davie EW (1986) Amino acid sequence of human histidine-rich glycoprotein derived from the nucleotide sequence of its cDNA. Biochemistry 25:2220-2225.

Kuroda K, Wake H, Mori S, Hinotsu S, Nishibori M, and Morimatsu H (2018) Decrease in histidine-rich glycoprotein as a novel biomarker to predict sepsis among systemic inflammatory response syndrome. Crit Care Med 46:570-576.

Mori S, Takahashi HK, Yamaoka K, Okamoto M, and Nishibori M (2003) High af finity binding of serum histidine-rich glycoprotein to nickel-nitrilotriacetic acid: the application to microquantification. Life Sci 73:93-102.

Nishibori M, Wake H, and Morimatsu H (2018) Histidine-rich glycoprotein as an excellent biomarker for sepsis and beyond. Crit Care 22:209.

Opal SM, Dellinger RP, Vincent JL, Masur H, and Angus DC (2014) The next generation of sepsis clinical trial designs: what is next after the demise of recombinant human activated protein C? Crit Care Med 42:1714-1721.

Papayannopoulos V, Metzler KD, Hakkim A, and Zychlinsky A (2010) Neutrophil elastase and myeloperoxidase regulate the formation of neutrophil extracellular traps. J Cell Biol 191:677-691.

Phillipson M and Kubes P (2011) The neutrophil in vascular inflammation. Nat Med 17:1381-1390.

Poon IK, Patel KK, Davis DS, Parish CR, and Hulett MD (2011) Histidine-rich glycoprotein: the Swiss Army knife of mammalian plasma. Blood 117:2093-2101.

Reinhart K, Bauer M, Riedemann NC, and Hartog CS (2012) New approaches to sepsis: molecular diagnostics and biomarkers. Clin Microbiol Rev 25:609-634.

Reinhart K, Daniels R, Kissoon N, Machado FR, Schachter RD, and Finfer S (2017) Recognizing sepsis as a global health priority - a WHO resolution. $N$ Engl J Med 377:414-417.

Remijsen Q, Vanden Berghe T, Wirawan E, Asselbergh B, Parthoens E, De Rycke R, Noppen S, Delforge M, Willems J, and Vandenabeele P (2011) Neutrophil extracellular trap cell death requires both autophagy and superoxide generation. Cell Res 21:290-304.

Rhee C, Dantes R, Epstein L, Murphy DJ, Seymour CW, Iwashyna TJ, Kadri SS, Angus DC, Danner RL, Fiore AE, et al.; CDC Prevention Epicenter Program (2017) Incidence and trends of sepsis in US hospitals using clinical vs claims data, 20092014. JAMA 318:1241-1249.

Singer M, Deutschman CS, Seymour CW, Shankar-Hari M, Annane D, Bauer M, Bellomo R, Bernard GR, Chiche JD, Coopersmith CM, et al. (2016) The third international consensus definitions for sepsis and septic shock (Sepsis-3). JAMA 315: 801-810.

Summers C, Rankin SM, Condliffe AM, Singh N, Peters AM, and Chilvers ER (2010) Neutrophil kinetics in health and disease. Trends Immunol 31:318-324.

Wake H, Mori S, Liu K, Morioka Y, Teshigawara K, Sakaguchi M, Kuroda K, Gao Y, Takahashi H, Ohtsuka A, et al. (2016) Histidine-rich glycoprotein prevents septic lethality through regulation of immunothrombosis and inflammation. EBioMedicine 9:180-194.

Zhong H, Wake H, Liu K, Gao Y, Teshigawara K, Sakaguchi M, Mori S, and Nishibori M (2018) Effects of Histidine-rich glycoprotein on erythrocyte aggregation and hemolysis: implications for a role under septic conditions. J Pharmacol Sci 136: 97-106.

Address correspondence to: Dr. Masahiro Nishibori, Department of Pharmacology, Graduate School of Medicine, Dentistry, and Pharmaceutical Sciences, Okayama University, 2-5-1 Shikata-cho, Kita-ku, Okayama 7008558, Japan. E-mail: mbori@md.okayama-u.ac.jp 